\title{
Getting to Know the Gut Microbial Diversity of Metropolitan Buenos Aires Inhabitants
}

\author{
Fiorella Sabrina Belforte1,2,3, Natalie Fernandez 4 , Francisco Tonín Monzón ${ }^{5}$, \\ Ayelén Daiana Rosso ${ }^{1,2}$, Sofía Quesada ${ }^{1,2}$, María Cecilia Cimolai ${ }^{1,2,3}$, Andrea Millán ${ }^{6}$, \\ Gloria Edith Cerrone ${ }^{6}$, Gustavo Daniel Frechtel ${ }^{6}$, Rémy Burcelin ${ }^{7,8}$, \\ Federico Coluccio Leskow ${ }^{2,3}$ and Alberto Penas-Steinhardt1,2,3,**
}

\begin{abstract}
${ }^{1}$ Laboratorio de Genómica Computacional, Departamento de Ciencias Básicas, Universidad Nacional de Luján, Luján, Argentina, ${ }^{2}$ Programa de Estudios de Comunicación y Señalización Inter-Reino (PECSI), Departamento de Ciencias Básicas, Universidad Nacional de Luján, Luján, Argentina, ${ }^{3}$ Consejo Nacional de Investigaciones Científicas y Técnicas (CONICET), CABA, Buenos Aires, Argentina, ${ }^{4}$ Icahn School of Medicine at Mount Sinai, New York, NY, United States, ${ }^{5}$ Centro de Investigación, Docencia y Extensión en Tecnologías de la Información y las Comunicaciones (CIDETIC), Universidad Nacional de Luján, Luján, Argentina, ${ }^{6}$ Laboratorio de Diabetes y Metabolismo, Instituto de Inmunología, Genética y Metabolismo, Universidad de Buenos Aires-CONICET, CABA, Buenos Aires, Argentina, ${ }^{7}$ Institut National de la Santé et de la Recherche Médicale (INSERM), Toulouse, France, ${ }^{8}$ Université Paul Sabatier (UPS), Unité Mixte de Recherche (UMR) 1048, Institut des Maladies Métaboliques et Cardiovasculaires (I2MC). Team 2: 'Intestinal Risk Factors, Diabetes, Dyslipidemia', Toulouse, France, ${ }^{9}$ Fundación H.A. Barceló, Instituto Universitario de Ciencias de la Salud, CABA, Buenos Aires, Argentina
\end{abstract}

Edited by:

M. Pilar Francino,

Fundación para el Fomento de la Investigación Sanitaria y Biomédica de la Comunitat Valenciana (FISABIO),

Spain

Reviewed by:

Harold Corke,

Shanghai Jiao Tong University, China

Federico Rey,

University of Wisconsin-Madison,

United States

*Correspondence: Alberto Penas-Steinhardt pufetin@gmail.com

Specialty section: This article was submitted to Microbial Symbioses, a section of the journal

Frontiers in Microbiology

Received: 07 November 2018 Accepted: 16 April 2019

Published: 21 May 2019

Citation:

Belforte FS, Fernandez N

Tonín Monzón F, Rosso AD,

Quesada S, Cimolai MC, Millán A, Cerrone GE, Frechtel GD, Burcelin $R$

Coluccio Leskow $F$ and Penas-Steinhardt A (2019) Getting to Know the Gut Microbial Diversity

of Metropolitan Buenos Aires Inhabitants. Front. Microbiol. 10:965.

doi: 10.3389/fmicb.2019.00965
In recent years, the field of immunology has been revolutionized by the growing understanding of the fundamental role of microbiota in the immune system function. The immune system has evolved to maintain a symbiotic relationship with these microbes. The aim of our study was to know in depth the uncharacterized metagenome of the Buenos Aires (BA) city population and its metropolitan area, being the second most populated agglomeration in the southern hemisphere. For this purpose, we evaluated 30 individuals (age: $35.23 \pm 8.26$ years and BMl: $23.91 \pm 3.4 \mathrm{~kg} / \mathrm{m}^{2}$ ), from the general population of BA. The hypervariable regions $\mathrm{V} 3-\mathrm{V} 4$ of the bacterial $16 \mathrm{~S}$ gene was sequenced by MiSeq-Illumina system, obtaining $47526 \pm 4718$ sequences/sample. The dominant phyla were Bacteroidetes, Firmicutes, Proteobacteria, Verrucomicrobia, and Actinobacteria. Additionally, we compared the microbiota of BA with other westernized populations (Santiago de Chile, Rosario-Argentina, United States-Human-microbiomeproject, Bologna-Italy) and the Hadza population of hunter-gatherers. The unweighted UniFrac clustered together all westernized populations, leaving the hunter-gatherer population from Hadza out. In particular, Santiago de Chile's population turns out to be the closest to BA's, principally due to the presence of Verrucomicrobiales of the genus Akkermansia. These microorganisms have been proposed as a hallmark of a healthy gut. Finally, westernized populations showed more abundant metabolism related KEEG pathways than hunter-gatherers, including carbohydrate metabolism (amino sugar and nucleotide sugar metabolism), amino acid metabolism (alanine, aspartate and glutamate metabolism), lipid metabolism, biosynthesis of secondary metabolites, and sulfur metabolism. These findings contribute to promote research and comparison of the microbiome in different human populations, in order to develop more efficient therapeutic strategies for the restoration of a healthy dialogue between host and environment. 


\section{INTRODUCTION}

Higher multicellular organisms, such as humans, can be considered meta-organisms constituted by the individual's own cells and their symbiotic commensal microbiota. With an estimated composition of 100 billion cells, human symbiotic microorganisms express 10 times more unique genes than those in the human genome (Ley et al., 2006). These complex communities of microorganisms, including bacteria, fungi, virus, and eukaryotic species not only enhance by several orders of magnitude the host's enzymatic capacity, but also play a key role in controlling many aspects of the individual's physiology.

In recent years, the field of immunology has been revolutionized by the growing understanding of the fundamental role of microbiota in the induction, training, and function of the host immune system (Belkaid and Hand, 2014). The immune system has evolved, to a large extent, as a means to maintain the symbiotic relationship between the host and these highly diverse and changing microbes. Under favorable conditions, the alliance between the immune system and the commensal microbiota allows the induction of protective responses against pathogens and the preservation of the regulatory pathways involved in the maintenance of tolerance to innocuous antigens (Chistiakov et al., 2014). However, profound changes in the environmental context could lead to the selection of a microbiota that lacks the resilience and diversity needed to establish balanced immune responses (Sommer et al., 2017). This suggests that environmental changes resulting from the modern lifestyle, such as antibiotic use, cesarean-section deliveries, excessive hygiene, and stress would account for the dramatic increase in autoimmune and chronic inflammatory disorders in geographical areas where the symbiotic relationship with the microbiota has been affected (Dominguez-Bello et al., 2010; Hall et al., 2017). Subclinical low-grade systemic inflammation has been associated with obesity, metabolic syndrome (MS) and type 2 diabetes mellitus (T2DM) (Schmidt et al., 2006). Several studies in animal models as well as in humans have shown that changes in the innate immune response are involved in the pathogenesis of these metabolic disorders (Hotamisligil, 2006; Cani et al., 2007). We have previously shown association between activity and expression levels of the toll-like receptor 4 (TLR4), a leucine-rich repeat molecule that plays a key role in the activation of innate immune response by recognizing conserved pathogen-associated molecular patterns such as lipopolysaccharides (LPS) and in metabolic disorders (Belforte et al., 2012; Penas-Steinhardt et al., 2012). The activation of TLR4 signaling induces up-regulation of inflammatory pathways related to the induction of insulin resistance. Others have highlighted the role of gut microbiota in the perpetuation of both insulin resistance and low-grade chronic inflammation (Petruzzelli and Moschetta, 2010), suggesting an alternative explanation for the increasing prevalence of insulin resistance related diseases.

There are several approaches under study to modulate the gut microbiota using external sources such as prebiotics, probiotics, antibiotics and fecal transplants (Balakrishnan and Taneja, 2018). Probiotics are live microbial species that modulate the gut microbiota in various ways: influencing immune homeostasis, regulating mucus secretion by gut epithelial cells, facilitating the production of a variety of nutrients such as vitamins, degrading toxic substances and producing antimicrobial compounds such as bacteriocins (Balakrishnan and Taneja, 2018). Consequently, regular probiotics consumption rapidly and reproducibly alters the human gut microbiome (David et al., 2014).

We are witnesses of an explosion of discoveries unveiling the role of gut microbial communities in health and disease states in humans (Garidou et al., 2015; Dickson, 2017). However, the normal composition of the microbiota of the human intestine is still under debate (van den Elsen et al., 2017). This provides a unique opportunity to develop a comprehensive exploration of human health from a multidisciplinary research strategy. Since Buenos Aires is the second most populated agglomeration in South America and the southern hemisphere, with a large genetic and cultural component of European immigration interacting with indigenous people, we propose to describe the fingerprint of its fecal microbiota. In this sense, it is fundamental to promote research defining the microbiome in different human populations to define future therapeutic strategies for the restoration of a healthy dialogue between host and environment, and thus to restore the health of the metaHuman organism.

\section{MATERIALS AND METHODS}

\section{Selection of Participants}

We evaluated 30 individuals (age: $35.23 \pm 8.26$ years and BMI: $23.91 \pm 3.4 \mathrm{~kg} / \mathrm{m}^{2}$ ), from the general population of the Autonomous City of Buenos Aires (BA) and its metropolitan area, Argentina. The recruitment was performed in the Endocrinology Service of Hospital de Clínicas "José de San Martín" at Universidad de Buenos Aires, where clinic and anthropometric measurements were performed, blood samples were collected, and stool samples were received. Fecal samples were collected in household collection tubes delivered by the researchers responsible for the project. All participants signed written informed consent approved by the Ethics Committee of Hospital Posadas, Buenos Aires (according to the bioethical principles of the Declaration of Helsinki), approved by the Teaching, Research and Bioethics Committee of the Hospital Posadas (Ref CEPIC 119 EUPeS1/16).

\section{Inclusion Criteria}

Volunteer individuals from the general population of the city of Buenos Aires.

\section{Exclusion Criteria}

Individuals that have received antibiotic therapy in the last month, extreme diets (macrobiotic, vegan), gastrointestinal surgery (gastrectomy, bariatric surgery, colostomy), pregnancy, neoplasia, patients in therapy of renal replacement, transplanted or HIV patients. 


\section{Phenotypic and Environmental Data}

Clinical and biochemical data were evaluated as well as anthropometric measurements of the participants. Demographic characteristics, presence of environmental factors (type of delivery, feeding, consumption of probiotics, alcohol, smoking, physical activity), medication used for treatment according to the case, and family history of chronic inflammatory pathologies, and the preexistence of emerging diseases such as Obesity, T2DM, Arterial Hypertension, Dyslipidemia among others, were established by anamnesis.

\section{Sample Collection and DNA Extraction}

During the interview participants were instructed in the collection method for stool sampling by receiving a standardized written protocol for the collection of approximately $5 \mathrm{~g}$ of stool in a sterile wide-mouth tube in DMSO/EDTA/saturated sodium chloride buffer (DESS buffer) (Gray et al., 2013). DESS Buffer has been shown to be highly effective in long-term DNA preservation at room temperature (Beknazarova et al., 2017). Tubes had a small spoon attached to the lid that facilitates the collection of the samples, which were suggested to be collected within $24 \mathrm{~h}$ prior to the next interview. Stool samples received were kept at $-80^{\circ} \mathrm{C}$ until use. For each sample, DNA extraction was performed from $200 \mathrm{mg}$ of feces using two different commercial kits following the instructions of the manufacturers: QIAamp DNA Stool Mini Kit (QIAGEN $\left.{ }^{\circledR}\right)$ and Quick-DNA Soil $\left(Z_{y m o}\right.$ Research $\left.^{\circledR}\right)$. Nucleic acid concentration and purity were determined by $1 \%$ agarose gel electrophoresis and spectrophotometry on a NanoDrop ND-1000 (NanoDrop Technologies, Wilmington, DE, United States).

\section{Sequencing of Bacterial 16S rRNA Fragments}

To amplify $16 \mathrm{~S}$ rRNA gene fragments, $30 \mathrm{ng}$ of purified DNA was used and the hypervariable regions V3 and V4 of the bacterial $16 \mathrm{~S}$ gene were amplified using Bakt_341F/Bakt_805R primers contained Illumina overhang adapter sequences. Sequencing was performed using a MiSeq sequencer (Illumina ${ }^{\circledR}$ ). Libraries were sequenced in paired-end mode, guaranteeing sequences of $\sim 550$ bp long and $\sim 100.000$ sequences per sample.

\section{Sequence Analysis and Comparison of Microbial Communities}

De-multiplexed reads were quality trimmed using Trimmomatic (V0.36) (Bolger et al., 2014). Sequences generated were analyzed using quantitative insights into microbial ecology (QIIME) version 1.9.1 software package (Caporaso et al., 2010) for the identification of operative taxonomic units (OTUs) as well as taxonomic allocation and statistical analysis. To this end, the sequences obtained were compared with those deposited in Greengenes 13_8 database (DeSantis et al., 2006). Chimeric sequences were filtered using VSEARCH (Rognes et al., 2016). Phylum, Class, Family, and Gender were assigned to each reading with an open_reference OTU picking process (with sortmerna_coverage of 80\%) (Kopylova et al., 2012). Additionally, to compare this dataset with other studies that targeted different $16 \mathrm{~S}$ regions, closed-reference strategy was used. In order to normalize the subsequent bioinformatic analysis raw sequences from different dataset were used. In particular, we compare our population with the following $16 \mathrm{~S}$ rRNA gene sequence data set: Healthy Cohort studyProduction Phase 2(PP2) of human microbiome project (HMP) SRP002860 (The Human Microbiome Project Consortium, 2012); Rosario-Argentina (Carbonetto et al., 2016); Hadza population from Tanzania (Schnorr et al., 2014); BolognaItalia (Schnorr et al., 2014) and Santiago-Chile (Schnorr et al., 2014; Fujio-Vejar et al., 2017). The sequences obtained in this study were forwarded and made available in Qiita database (ID 11839) and SRA: PRJNA503303. To account for multiple comparisons, $p$-values were adjusted by Benjamini-Hochberg FDR correction, applied separately at each taxonomic rank for a given comparison (Benjamini and Hochberg, 1995). To study microbial communities in different sample groups we used Unifrac algorithm (Lozupone and Knight, 2015), specially designed to describe and compare microbial communities based on massive sequencing of phylogenetic marker genes. In order to compare this dataset with other studies that targeted different $16 \mathrm{~S}$ regions closed_reference strategy was used. Differences on beta diversity were assessed using ADONIS. Ellipses drawings for weighted UniFrac and unweighted UniFrac were performed using cov.dellipse from metRology R package.

Putative functional genes were predicted using PICRUSt (Phylogenetic Investigation of Communities by Reconstruction of Unobserved States) (Langille et al., 2013), which applies 16S rRNA gene to predict the abundance of functional genes by matching sample OTUs with reference genomes. In order to compare the relative abundance of the different taxa between groups, we performed linear discriminant analysis (LDA) effect implemented in LEfSe (Segata et al., 2011).

\section{RESULTS}

\section{Buenos Aires General Population Data}

Buenos Aires and its metropolitan area constitute a megalopolis, being the second most populated agglomeration in South America and the southern hemisphere with 12.806 .866 of inhabitants. According to the last census (2010), the average age of the Autonomous City of Buenos Aires (BA) is 39.66 years. As reported by the "Third National Survey of Risk Factors for Non-transmissible Diseases" (NSRF) of the National Ministry of Health performed in 2013 (Tercera encuesta nacional de factores de riesgo para enfermedades no transmisibles, 2013), the average daily servings of fruits or vegetables consumed per person in the general population of BA was 2.1, being below the amount recommended by WHO (five daily servings of fruits and/or vegetables). The highest averages of daily fruit or vegetable consumption were recorded in the oldest age group (2.4), in the highest education level (2.1) and higher income level. In addition, the prevalence of overweight individuals was 32\% (IC 95\% 27.6 36.8), the prevalence of high cholesterol among those who were once controlled (population 18 years and older) was $27.9 \%$ (IC $95 \% 24.0-32.1$ ), and $8.2 \%$ marked the prevalence of diabetes in the total adult population (IC $95 \% 6.4-10.5$ ). 
We evaluated 30 individuals (Female/male: 15/15; age: $35.23 \pm 8.26$ years and BMI: $23.91 \pm 3.4 \mathrm{~kg} / \mathrm{m}^{2}$ ), from the general population of BA (Table 1 ).

\section{Samples Collection and Storage}

Considering that several methods could be used for stool collection and storage, we standardized sample collection and storage protocol prior processing. In our population based study, participants were instructed in a home collection method for stool sampling, proved to be as effective as freshly collected native stool (Schultze et al., 2014). This strategy comes from the preference of most participants who do not agree to collect the stool samples elsewhere. Therefore, each participant received a written protocol for easy stool collection, consisting in introducing approximately $5 \mathrm{~g}$ of stool in a sterile widemouth tube with DESS buffer (Gray et al., 2013). This solution preserves the samples both at room temperature and in subzero conditions, avoiding freezing and cell damage. Additionally, DESS buffer is a non-proprietary solution that has been used for DNA preservation (Gaither et al., 2010) as it guarantees long term storage at room temperature (up to 24 weeks), and has been used to preserve bacterial DNA from coral mucus swabs for up to 4.5 months (May, 2011). Additionally, given that it is known that freezing and thawing the samples could generate OTUs identification bias (Fouhy et al., 2015), this buffer represents an efficient alternative strategy for adequate sampling and preservation until processing.

\section{DNA Extraction Method}

DNA isolation protocol and the targeted 16S rRNA gene region sequence chosen for analysis have been shown to be critical for gut microbiota assessment (Voigt et al., 2015), limiting the ability to compare different human microbiome datasets. In other to evaluate possible biases in the $16 \mathrm{~S}$ profiles related to DNA extraction method, we compare QIAamp DNA Stool Mini (QG) and Quick-DNA Soil (ZR) commercial kits. DNA extraction was performed from $200 \mathrm{mg}$ of feces using both QG and ZR for each sample. Fecal DNA extraction using QC and ZR yielded an average of $49.59 \pm 21.41$ and $24.85 \pm 20.75 \mu \mathrm{g} / \mathrm{mL}$ DNA, respectively. The hypervariable regions V3-V4 of bacterial 16S gene was sequenced using MiSeq-Illumina system, and an average of $47526 \pm 4718$ sequences per sample were obtained.

TABLE 1 | Anthropometric and biochemical data of the 30 volunteers.

\begin{tabular}{lrr}
\hline & Mean & SD \\
\hline Female/male & $15 / 1$ & \\
Age (years) & 35.23 & 8.26 \\
Weight $(\mathrm{kg})$ & 69.48 & 12.82 \\
Height $(\mathrm{m})$ & 170.17 & 9.73 \\
Body mass index $\left(\mathrm{kg} / \mathrm{m}^{2}\right)$ & 23.91 & 3.40 \\
Glycemia $(\mathrm{mg} / \mathrm{dl})$ & 80.48 & 11.33 \\
Triglycerides $(\mathrm{mg} / \mathrm{dl})$ & 75.24 & 20.48 \\
Total cholesterol $(\mathrm{mg} / \mathrm{dl})$ & 168.24 & 29.80 \\
High density lipoproteins $(\mathrm{mg} / \mathrm{dl})$ & 55.10 & 17.06 \\
Low density lipoproteins $(\mathrm{mg} / \mathrm{dl})$ & 168.23 & 29.80
\end{tabular}

Rarefaction plots reach an asymptotic state, indicating that the sequence depth was sufficient to represent the bacterial community richness and diversity by both methods (data not shown). In this sense, when we compare alpha diversity (Chao1 index) based on a two-sample $t$-test, there are not significant differences between QG and ZR kits $(p=0.069)$. We also found no significant differences when comparing beta diversity between different purification kits, $p=0.97$ for weighted UniFrac and unweighted UniFrac $p=0.294$ (ADONIS).

Comparing the principal phyla detected using both DNA extraction Kits, we found that the choice of DNA extraction method has an impact on the observed community structure. Using the QG kit, the dominant phyla were Bacteroidetes $(47.7 \pm 8.9 \%)$, Firmicutes (37.2 $\pm 8.45 \%)$, Proteobacteria $(8.5 \pm 6.7 \%)$, Verrucomicrobia $(2.5 \pm 2.8 \%)$ and Actinobacteria $(1.3 \pm 2.2 \%)$, while the principal phyla found using ZR kit were Firmicutes $(45.5 \pm 8.8 \%)$, and Bacteroidetes (40.0 $\pm 7.8 \%)$, followed by Proteobacteria (6.1 $\pm 5.4 \%)$, Verrucomicrobia $(2.5 \pm 3.0 \%)$, and Actinobacteria $(3.1 \pm 2.2 \%)$. In this sense, QG protocol, without the bead-beating step, resulted in a significantly low abundance in Gram-positive phyla, Firmicutes $(p=0.015)$; Actinobacteria $\left(p=9.29 \times 10^{-5}\right)$ and overestimated Bacteroidetes phyla $(p=0.023)$ FDR adjusted $p$-values. These results, in accordance with previous studies (Guo and Zhang, 2013; Wesolowska-Andersen et al., 2014), show that DNA extraction method has an impact on the observed community structure. In particular, the first step of DNA extraction beadbeating disruption and/or lysis of the bacterial membranes can be expected to be biased for specific bacterial taxa due to differences in cell wall structure and integrity.

Since several studies have proposed the Firmicutes to Bacteroidetes ratio (F/B:ratio) as a gauge of short-chain fatty acids (SCFA) production and the overall gut microbiota balance any bias in these parameters must be taken into account (Rodríguez-Carrio et al., 2017; Garcia-Mantrana et al., 2018). In particular, QG protocol showed an increase in the abundance of Bacteroidetes phylum, main acetate and propionate producers, and a decrease in Firmicutes butyrate-producers (Macfarlane and Macfarlane, 2003). It is important to remark that these changes impact notably the F/B: ratio, being 0.77 for QG kit vs. 1.13 for $\mathrm{ZR}$ one. However, it is not possible to determine which DNA extraction method best represents the overall microbial community.

\section{Analysis of BA Microbiota}

Our data is consistent with previous observations showing that the regular intake of yogurt and other fermented products promotes a healthy gut microbiota (Lisko et al., 2017; Marco et al., 2017; Suzuki et al., 2017). In this sense we found significant differences in alpha diversity Chaol index $(p=0.012)$ when comparing microbial diversity between subjects that habitually consume probiotics with those who do not (considering both extraction kits).

To further analyze our results, we compared the microbiota of BA with other westernized populations (Santiago de Chile, Rosario-Argentina, United States-Human-microbiome-project, Bologna-Italy) and the Hadza population of hunter-gatherers 
TABLE 2 | Data-sets included in the analysis.

\begin{tabular}{|c|c|c|c|c|c|c|c|}
\hline Platform & Country & City & Purification_kit & Region & Center_name & Habitat & Publication \\
\hline $\begin{array}{l}454 \text { LifeSciences- } \\
\text { Roche }\end{array}$ & Tanzania & $\begin{array}{l}\text { Tanzania-Lake } \\
\text { Eyasi }\end{array}$ & $\begin{array}{l}\text { QIAmp DNA Stool } \\
\text { Mini Kit (Qiagen) }\end{array}$ & V4 & $\begin{array}{l}\text { Metabolism } \\
\text { Anthropometry } \\
\text { and Nutrition } \\
\text { Laboratory } \\
\text { Department of } \\
\text { Anthropology } \\
\text { University of } \\
\text { Nevada. }\end{array}$ & Hunter-Gathe rer & $\begin{array}{l}\text { Gut microbiome of the } \\
\text { Hadza hunter-gatherer } \\
\text { s_doi: } 10.1038 / \mathrm{n} \\
\text { comms } 4654\end{array}$ \\
\hline $\begin{array}{l}454 \text { LifeSciences- } \\
\text { Roche }\end{array}$ & Italy & Italy-Bologna & $\begin{array}{l}\text { QIAmp DNA Stool } \\
\text { Mini Kit (Qiagen) }\end{array}$ & V4 & $\begin{array}{l}\text { Metabolism } \\
\text { Anthropometry } \\
\text { and Nutrition } \\
\text { Laboratory } \\
\text { Department of } \\
\text { Anthropology } \\
\text { University of } \\
\text { Nevada. }\end{array}$ & Westernized & $\begin{array}{l}\text { Gut microbiome of the } \\
\text { Hadza hunter-gatherer } \\
\text { s_doi: } 10.1038 / \mathrm{n} \\
\text { comms } 4681\end{array}$ \\
\hline $\begin{array}{l}\text { MiSeqSytem } \\
\text {-Illumina }\end{array}$ & Argentina & $\begin{array}{l}\text { Argentina- } \\
\text { Buenos_Aires }\end{array}$ & $\begin{array}{l}\text { QIAmp DNA Stool } \\
\text { Mini Kit (Qiagen) } \\
\text { and Quick-DNA } \\
\text { Soil (Zymo } \\
\text { Research). }\end{array}$ & V3-V4 & GeC-UNLU & Westernized & Buenos Aires microbiome \\
\hline $\begin{array}{l}\text { MiSeqSytem } \\
\text {-Illumina }\end{array}$ & Chile & Chile-Santiago & $\begin{array}{l}\text { QIAmp DNA Stool } \\
\text { Mini Kit (Qiagen) }\end{array}$ & V3-V4 & $\begin{array}{l}\text { Centro de } \\
\text { Genética y } \\
\text { Genómica }\end{array}$ & Westernized & $\begin{array}{l}\text { The Gut Microbiota of } \\
\text { Healthy Chilean Subjects } \\
\text { Reveals a High Abundance } \\
\text { of the Phylum } \\
\text { Verrucomicrobi a: } \\
\text { https://doi.org/10.3389/ } \\
\text { fmicb.2017.01221 }\end{array}$ \\
\hline $\begin{array}{l}454 \text { LifeSciences- } \\
\text { Roche }\end{array}$ & Argentina & Argentina-Rosario & $\begin{array}{l}\text { QIAmp DNA Stool } \\
\text { Mini Kit (Qiagen) }\end{array}$ & $V_{1}-V_{3}$ & INDEAR & Westernized & $\begin{array}{l}\text { Human Microbiota of the } \\
\text { Argentine Population- A } \\
\text { Pilot Study: https://doi.org/ } \\
\text { 10.3389/fmicb.2016.00051 }\end{array}$ \\
\hline $\begin{array}{l}454 \text { LifeSciences- } \\
\text { Roche }\end{array}$ & United States & United States & $\begin{array}{l}\text { MoBio Powersoil } \\
\text { Kit }\end{array}$ & V3-V5 & $\begin{array}{l}\text { Human } \\
\text { Microbiome } \\
\text { Project (HMP) }\end{array}$ & Westernized & $\begin{array}{l}\text { Healthy Cohort } \\
\text { study:Productio n Phase } \\
\text { 2(PP2)-SRP002 } 860\end{array}$ \\
\hline
\end{tabular}

(Table 2). To understand differences in the composition of microbial communities' diversity among environments was compared. Beta diversity (considering weighted and unweighted UniFrac distances) was analyzed in order to compare the differences between microbial compositions among different populations (Figure 1). Differences on beta diversity values between habitats, countries, cities, $16 \mathrm{~S}$ region amplified, purification kit employed, and platform were evaluated (ADONIS). Although all the comparisons were statistically significant ( $p<0.001$ ), the $R^{2}$-value indicates that approximately $43 \%$ (weighted) and 23\% (unweighted) of the variation effect is produced by the city of origin, being the most important variable studied. The unweighted UniFrac (a qualitative measure that estimates the distance between two communities) clustered the westernized populations together, leaving the hunter-gatherer population from Hadza out. In particular, Santiago de Chile's population turns out to be the closest to BA's.

It is relevant to highlight that the microbiota of $\mathrm{BA}$ subjects stands out for the presence of Verrucomicrobia of the genus Akkermansia (1.2\%). The mucus-degrading bacterium Akkermansia muciniphila is the only identified member of this genus and was also found in the Chilean population (FujioVejar et al., 2017). This microorganism has been proposed by others as a hallmark of a healthy gut due to its antiinflammatory and immunostimulant properties and its ability to improve gut barrier function, insulin sensitivity and endotoxemia (Collado et al., 2007; Bland, 2016). However, some studies report the induction of pro-inflammatory responses triggered by Akkermansia contributing to an increase of inflammation during infection in Multiple Sclerosis patients (Cekanaviciute et al., 2017). As proposed by Fujio-Vejar et al., these results could serve as a baseline for the characterization of dysbiosis associated with various diseases (Fujio-Vejar et al., 2017; Figure 2).

Putative functional genes were predicted using PICRUSt which applies 16S rRNA gene to predict the abundance of functional KEEG pathways. The LDA effective size (LEfSe) was performed to identify KEEG pathways with statistically differential abundance between hunter-gatherers and westernized population. The results show that metabolism related pathways were more abundant in westernized populations than in hunter-gatherers. In particular, carbohydrate metabolism (amino sugar and nucleotide sugar metabolism), amino 

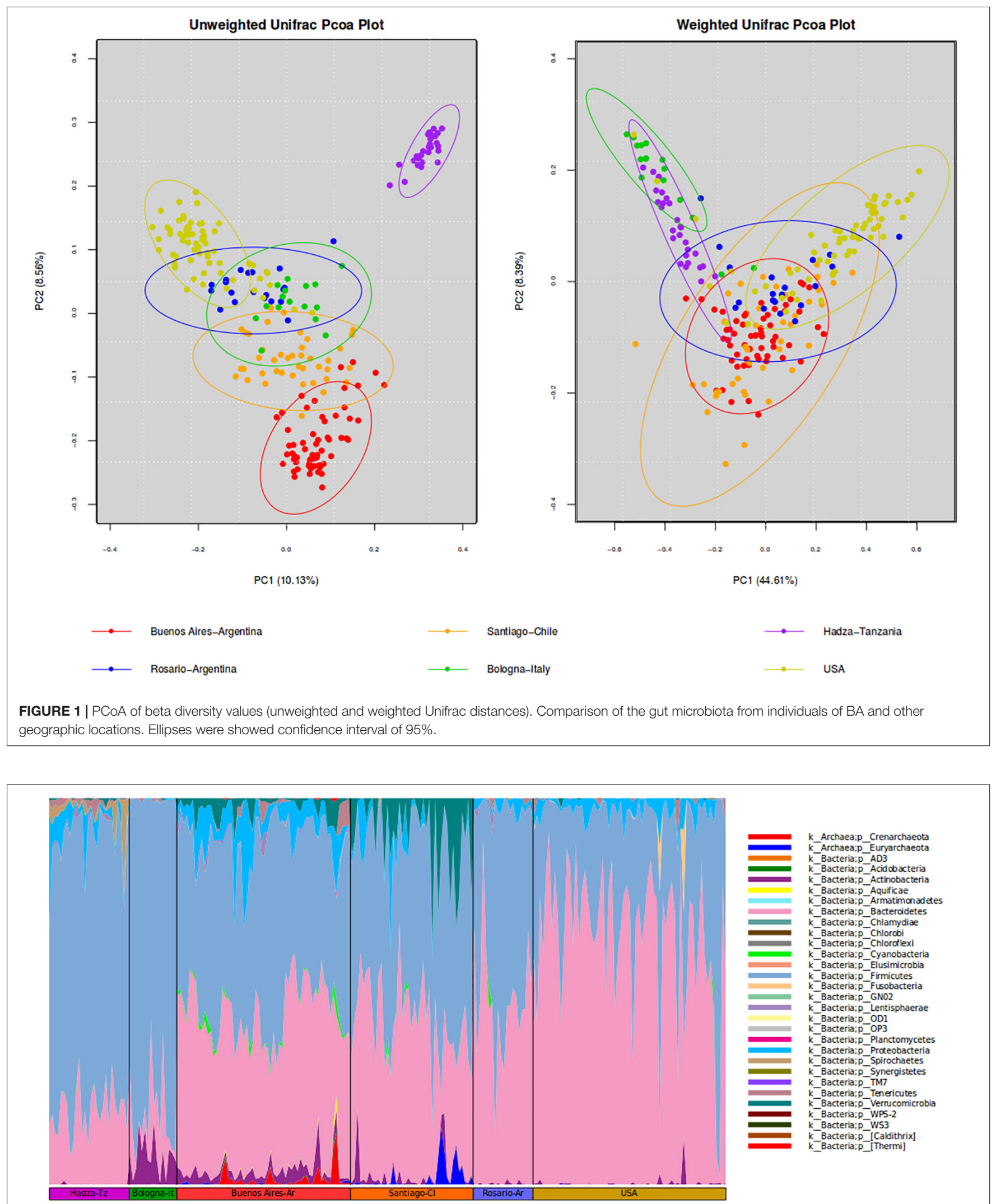

FIGURE 2 | Community composition of gut microbiome from each geographic location shown at the phylum level. 


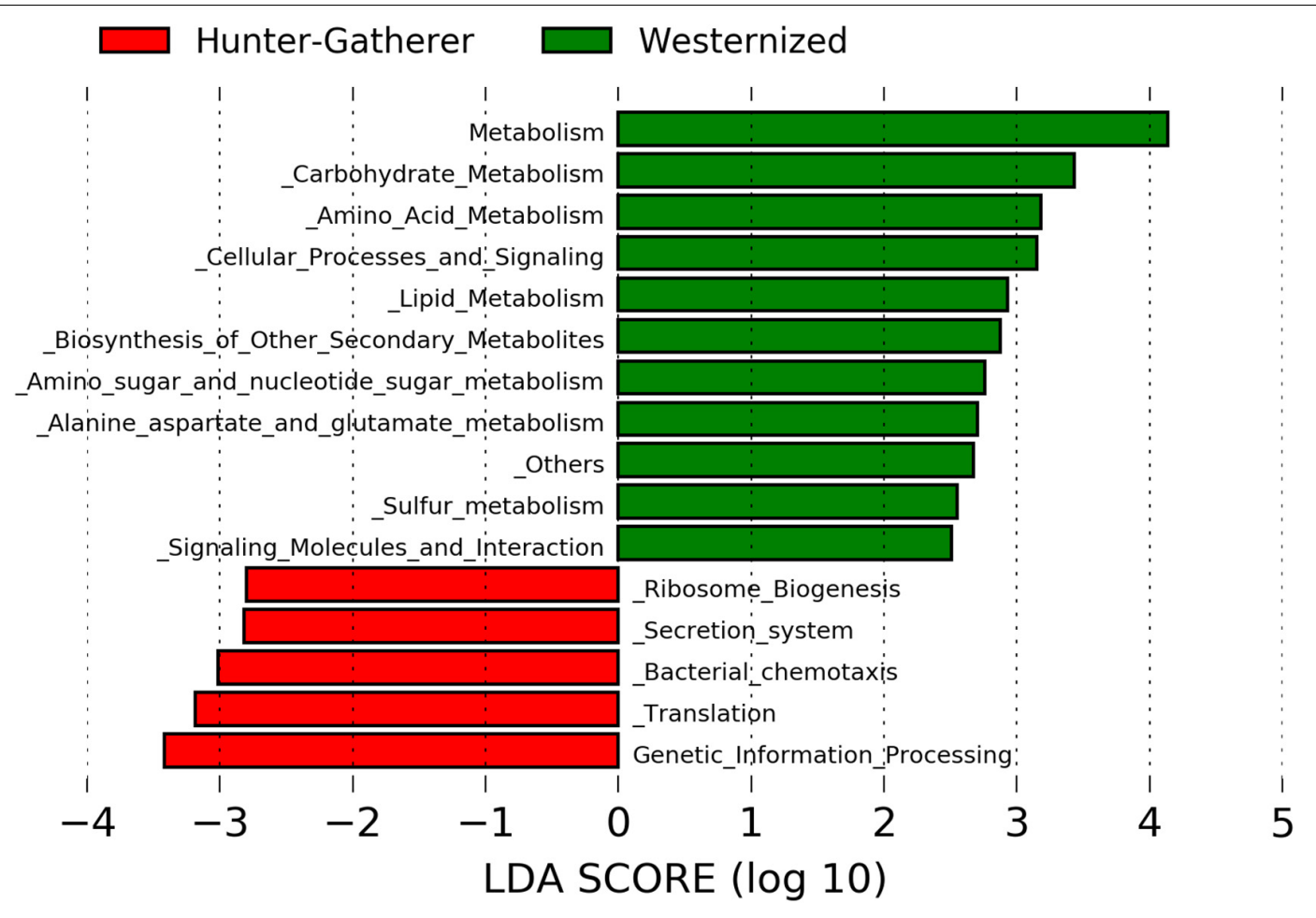

FIGURE 3 | Linear discriminant analysis (LDA) effect size (LEfSe) analysis of the differentially abundant KEEG pathways in hunter-gatherers vs. westernized population. The threshold of the logarithmic LDA score was 2.0.

acid metabolism (alanine, aspartate and glutamate metabolism), lipid metabolism, biosynthesis of secondary metabolites, and sulfur metabolism showed a LDA score greater than 2.0 (Figure 3).

\section{DISCUSSION}

The intestine harbors a sophisticated ecosystem of microbial communities, exerting vital metabolic functions that contribute to the recovery of nutrients and energy from non-digestible substrates. Microbial colonization is essential for the normal development of the immune system, regulating the homeostasis between environmental antigenic load and immune response. In susceptible individuals, imbalance could result into pathologies of immune dysregulation, including chronic inflammatory bowel diseases and metabolic syndrome, in which the immune system overreacts to non-harmful microbial antigens (Ananthakrishnan et al., 2017; Burcelin, 2017). Although there are new molecular technologies, the normal composition of the microbiota of the human intestine is still under debate (van den Elsen et al., 2017). Many environmental factors, such as geographic localization, household characteristics and circumstances as delivery method, antibiotic use, breastfeeding or diet in the early life, are conditioning factors for microbial colonization of the intestine. In permissive genetic backgrounds, environmental reprogramming of microbiota can trigger conditions for the establishment of an imbalanced immune response (Ussar et al., 2015).
The number of public human metagenomes shows a marked bias toward the north hemisphere. Analyzing the total metagenomic data available today, the northern hemisphere outnumbers that from the south by 200 times. Since Buenos Aires is the second most populated agglomeration in South America and the southern hemisphere, with a large genetic and cultural component of European immigration interacting with indigenous people, we propose to describe the fingerprint of its fecal microbiota. Given the hypothesis that there are differences between the intestinal microbiota composition of healthy and diseased subjects, we proposed to draw a baseline study of the uncharacterized gut microbiota from the general population of BA for future comparisons with any further studies.

During the interviews, participants were instructed in the home collection method of stool sampling, which was suggested to be collected within the $24 \mathrm{~h}$ prior to the next interview. Stool samples were remitted and stored at $-80^{\circ} \mathrm{C}$ until use. In order to prevent changes in fecal microbiota, $16 \mathrm{~S}$ gene sequencing profiles related to ambient temperature (Roesch et al., 2009; Carroll et al., 2012) or sub-zero storage conditions (Bahl et al., 2012; Fouhy et al., 2015) sampling was performed in a sterile wide-mouth tube with DESS buffer, since performing the nucleic acid extraction of samples immediately after collection was impractical. DESS buffer showed to be particularly efficient for sample transfer and storage at $-80^{\circ} \mathrm{C}$ until processing, and it allowed the adequate conservation of the samples. 
DNA extraction could also add to methodological biases in PCR amplification due to the presence of contaminants that could inhibit the reaction or the efficiency in DNA purification. Additionally, disruption and/or lysis of the bacterial membranes could be another source of bias, as it may favor the presence of specific bacterial taxa due to differences in cell wall structure and integrity (Sinha et al., 2016; Vogtmann et al., 2017). We have chosen two worldwide used commercial kits in order to evaluate microbiota profile sampling. Indeed, we observed differences in two of the most abundant phyla, Firmicutes and Bacteroidetes, which were differently affected, showing the effect of the extraction methods between Gram-positive and Gram-negative bacterial membranes. Interestingly, when we compare alpha diversity no significant differences between kits were observed. We also found no significant differences when comparing beta diversity, for weighted UniFrac and unweighted UniFrac, between purification methods. Although principal phyla were detected using both kits, we found that the choice of DNA extraction method has an impact on the observed community structure. In particular, these notably alter the $\mathrm{F} / \mathrm{B}$ ratio, not allowing determining which DNA extraction method is the best to represent the microbial community. In addition, when we compare the microbiota of BA with other populations, we observed differences on beta diversity values between habitats, countries, cities, 16S region amplified, Purification Kit employed and Platform. Although all the comparisons were statistically significant, the $R^{2}$-value indicates that approximately $43 \%$ (weighted) and $23 \%$ (unweighted) of the variation effect is produced by the city of origin, being the most important variable studied. However, when taxa abundance, such as $\mathrm{F} / \mathrm{B}$ ratio, is analyzed between different studies the purification method must be taken into account. In this sense, future gold standard purification method should be developed to sort this bias to allow accurate conclusions.

In order to evaluate similarities and differences within other populations, we compare our microbiome with other westernized populations and the Hadza population of hunter-gatherers. The unweighted UniFrac, that estimate the distance between two communities, clustered together all westernized populations leaving the hunter-gatherer population from Hadza out. This Qualitative measure which uses only the presence/absence of data is most informative when communities differ primarily by which microorganism can live in them (Lozupone et al., 2007). Additionally, unweighted UniFrac can better detect effects of different founding populations, such as the source of bacteria that first colonize the gut of newborns and the effects of restrictive factors for microbial growth. In contrast, quantitative measures that account for the relative abundance of microbial lineages can reveal the effects of more transient factors.

In this sense, the microbiota of BA subjects stands out for the presence of the mucus-degrading bacterium Akkermansia muciniphila, the only identified member of its genus. This microorganism, also found in the Chilean population, has been proposed as a hallmark of a healthy gut due to its antiinflammatory and immunostimulant properties and its ability to improve gut barrier function, insulin sensitivity and endotoxemia (Collado et al., 2007; Fujio-Vejar et al., 2017).
Finally, westernized populations showed more abundant metabolism related KEEG pathways than hunter-gatherers, including carbohydrate metabolism (amino sugar and nucleotide sugar metabolism), amino acid metabolism (alanine, aspartate and glutamate metabolism), lipid metabolism, biosynthesis of secondary metabolites and sulfur metabolism. This could be due to feeding habits since Hadza diet consists only of wild foods that fall into five main categories: meat, honey, baobab, berries and tubers (Schnorr et al., 2014).

Environmental factors, such as lifestyle, diet, the use of antibiotics, and the host genotype affects the human microbiome. It is important to point out that most of the available studies evaluate European, Asian, and North American populations that significantly differ both in the genetic background and in diverse environmental factors with BA population. On the contrary, very little is known about the microbiome of the Argentinean population, in particular there is currently only a pilot study of the population of Rosario city, Santa Fe (Carbonetto et al., 2016). It was then essential to know in depth the characteristic metagenome of the residents of BA and its metropolitan area which constitute a megalopolis, being the second most populated agglomeration in South America and the southern hemisphere with 12.806 .866 of inhabitants.

In this sense, it is crucial to identify specific groups of the intestinal microbiota that could affect the susceptibility and/or severity of disease by stimulating chronic inflammatory responses (Surana and Kasper, 2017). Diversity among environments, genotypes and, therefore, microbial factors of the intestinal flora between human populations make it difficult to find ubiquitous biomarkers worldwide. Knowing the microbiota composition of the general BA population provides a framework to have a local reference that will allow establishing future correlations between health and disease. This is essential, not only for evaluating changes in the gut microbiota regarding different social habits and clinical features, but also to contribute to the development of personalized nutritional and pharmacological strategies to promote a healthy intestinal flora.

\section{ETHICS STATEMENT}

This study was carried out in accordance with the recommendations of the Academic and Bioethics Committee of the National University of Luján with written information consent from all subjects. All subjects gave written informed consent in accordance with the Declaration of Helsinki. The protocol was approved by the Academic and Bioethics Committee of the National University of Luján (Ref TRI-LUJ: 0005667/2018).

\section{AUTHOR CONTRIBUTIONS}

FB and AP-S designed the study. NF, GC, and GF performed the recruitment of the volunteers. NF, AR, SQ, and AM collected the stool samples and the extraction of fecal bacterial DNA. FB, FTM, and AP-S processed the raw sequences and performed the 
bioinformatic and statistical analysis. FB, MC, RB, FCL, and AP-S analyzed the results and wrote the manuscript.

\section{FUNDING}

This work was supported by Mount Sinai International Exchange Program for Minority Students (Grant \#: 5T37MD001452-14) from the National Center on Minority Health and Health Disparities of the United States National Institutes of Health, Fundación Barceló res. HCS $\mathrm{N}^{\circ}$ 6642, PICT-2017-2406, Fundación Florencio Fiorini and Asociación Médica Argentina 2017, UNLu CD-CB NN 210/17, UNLu CD-CB NN ${ }^{\circ} 435 / 16$, and UNLu CD-CB $\mathrm{NN}^{\circ} 421 / 17$. The funders had no role in

\section{REFERENCES}

Ananthakrishnan, A. N., Bernstein, C. N., Iliopoulos, D., Macpherson, A., Neurath, M. F., Ali, R. A. R., et al. (2017). Environmental triggers in IBD: a review of progress and evidence. Nat. Rev. Gastroenterol. Hepatol. 15, 39-49. doi: 10.1038/nrgastro.2017.136

Bahl, M. I., Bergström, A., and Licht, T. R. (2012). Freezing fecal samples prior to DNA extraction affects the Firmicutes to Bacteroidetes ratio determined by downstream quantitative PCR analysis. FEMS Microbiol. Lett. 329, 193-197. doi: 10.1111/j.1574-6968.2012.02523.x

Balakrishnan, B., and Taneja, V. (2018). Microbial modulation of the gut microbiome for treating autoimmune diseases. Expert Rev. Gastroenterol. Hepatol. 12, 985-996. doi: 10.1080/17474124.2018.1517044

Beknazarova, M., Millsteed, S., Robertson, G., Whiley, H., and Ross, K. (2017). Validation of DESS as a DNA preservation method for the detection of strongyloides spp. in Canine Feces. Int. J. Environ. Res. Public Health 14:E624. doi: 10.3390/ijerph14060624

Belforte, F. S., Coluccio Leskow, F., Poskus, E., and Penas Steinhardt, A. (2012). Toll-like receptor 4 D299G polymorphism in metabolic disorders: a metaanalysis. Mol. Biol. Rep. 40, 3015-3020. doi: 10.1007/s11033-012-2374-5

Belkaid, Y., and Hand, T. W. (2014). Role of the microbiota in immunity and inflammation. Cell. 157, 121-141. doi: 10.1016/j.cell.2014.03.011

Benjamini, Y., and Hochberg, Y. (1995). Controlling the false discovery rate: a practical and powerful approach to multiple testing. J. R. Stat. Soc. Ser. B Stat. Methodol. 57, 289-300. doi: 10.1111/j.2517-6161.1995.tb02031.x

Bland, J. (2016). Intestinal microbiome, and medical nutrition therapy. Integr. Med. $15,14-16$.

Bolger, A. M., Lohse, M., and Usadel, B. (2014). Trimmomatic: a flexible trimmer for Illumina sequence data. Bioinformatics 30, 2114-2120. doi: 10 . 1093/bioinformatics/btu170

Burcelin, R. (2017). [Gut microbiota and immune crosstalk in metabolic disease]. Biol. Aujourdhui 211, 1-18.

Cani, P. D., Amar, J., Iglesias, M. A., Poggi, M., Knauf, C., Bastelica, D., et al. (2007). Metabolic endotoxemia initiates obesity and insulin resistance. Diabetes $56,1761-1772$.

Caporaso, J. G., Kuczynski, J., Stombaugh, J., Bittinger, K., Bushman, F. D., Costello, E. K., et al. (2010). QIIME allows analysis of high-throughput community sequencing data. Nat. Methods 7, 335-336.

Carbonetto, B., Fabbro, M. C., Sciara, M., Seravalle, A., Méjico, G., Revale, S., et al. (2016). Human microbiota of the argentine population- a pilot study. Front. Microbiol. 7:51. doi: 10.3389/fmicb.2016.00051

Carroll, I. M., Ringel-Kulka, T., Siddle, J. P., Klaenhammer, T. R., and Ringel, Y. (2012). Characterization of the fecal microbiota using high-throughput sequencing reveals a stable microbial community during storage. PLoS One 7:e46953. doi: 10.1371/journal.pone.0046953

Cekanaviciute, E., Yoo, B. B., Runia, T. F., Debelius, J. W., Singh, S., Nelson, C. A., et al. (2017). Gut bacteria from multiple sclerosis patients modulate human $\mathrm{T}$ cells and exacerbate symptoms in mouse models. Proc. Natl. Acad. Sci. U.S.A. 144, 10713-10718. doi: 10.1073/pnas.1711235114 study design, data collection and analysis, decision to publish, or preparation of the manuscript.

\section{ACKNOWLEDGMENTS}

The authors thank Centro de Investigación, Docencia y Extensión en Tecnologías de la Información y las Comunicaciones (CIDETIC, http:/cidetic.unlu.edu.ar/), Universidad Nacional de Luján, Luján, Argentina for human and computational resources, Edgardo Poskus, Silvina Valdez, Luciano Guerra, and Natalia I. Faccinetti for their topical and intellectual discussions, and the Endocrinology Service Staff of Hospital de Clínicas "José de San Martín", Buenos Aires, Argentina.

Chistiakov, D. A., Bobryshev, Y. V., Kozarov, E., Sobenin, I. A., and Orekhov, A. N. (2014). Intestinal mucosal tolerance and impact of gut microbiota to mucosal tolerance. Front. Microbiol. 5:781. doi: 10.3389/fmicb.2014.00781

Collado, M. C., Derrien, M., Isolauri, E., de Vos, W. M., and Salminen, S. (2007). Intestinal integrity and Akkermansia muciniphila, a mucin-degrading member of the intestinal microbiota present in infants, adults, and the elderly. Appl. Environ. Microbiol. 73, 7767-7770. doi: 10.1128/aem.01477-07

David, L. A., Maurice, C. F., Carmody, R. N., Gootenberg, D. B., Button, J. E., Wolfe, B. E., et al. (2014). Diet rapidly and reproducibly alters the human gut microbiome. Nature 505, 559-563. doi: 10.1038/nature12820

DeSantis, T. Z., Hugenholtz, P., Larsen, N., Rojas, M., Brodie, E. L., Keller, K., et al. (2006). Greengenes, a chimera-checked 16S rRNA gene database and workbench compatible with ARB. Appl. Environ. Microbiol. 72, 5069-5072. doi: 10.1128/aem.03006-05

Dickson, I. (2017). Gut microbiota: diagnosing IBD with the gut microbiome. Nat. Rev. Gastroenterol. Hepatol. 14:195. doi: 10.1038/nrgastro.2017.25

Dominguez-Bello, M. G., Costello, E. K., Contreras, M., Magris, M., Hidalgo, G., Fierer, N., et al. (2010). Delivery mode shapes the acquisition and structure of the initial microbiota across multiple body habitats in newborns. Proc. Natl. Acad. Sci. U.S.A. 107, 11971-11975. doi: 10.1073/pnas.10026 01107

Fouhy, F., Deane, J., Rea, M. C., O’Sullivan, Ó, Paul Ross, R., O’Callaghan, G., et al. (2015). The effects of freezing on faecal microbiota as determined using miseq sequencing and culture-based investigations. PLoS One 10:e0119355. doi: 10.1371/journal.pone.0119355

Fujio-Vejar, S., Vasquez, Y., Morales, P., Magne, F., Vera-Wolf, P., Ugalde, J. A., et al. (2017). The gut microbiota of healthy chilean subjects reveals a high abundance of the phylum verrucomicrobia. Front. Microbiol. 8:1221. doi: 10. 3389/fmicb.2017.01221

Gaither, M. R., Szabó, Z., Crepeau, M. W., Bird, C. E., and Toonen, R. J. (2010). Preservation of corals in salt-saturated DMSO buffer is superior to ethanol for PCR experiments. Coral Reefs 30, 329-333. doi: 10.1007/s00338-010-0687-1

Garcia-Mantrana, I., Selma-Royo, M., Alcantara, C., and Collado, M. C. (2018). Shifts on gut microbiota associated to mediterranean diet adherence and specific dietary intakes on general adult population. Front. Microbiol. 9:890. doi: 10.3389/fmicb.2018.00890

Garidou, L., Pomié, C., Klopp, P., Waget, A., Charpentier, J., Aloulou, M., et al. (2015). The gut microbiota regulates intestinal CD4 T cells expressing ROR $\gamma \mathrm{t}$ and controls metabolic disease. Cell Metab. 22, 100-112. doi: 10.1016/j.cmet. 2015.06.001

Gray, M. A., Pratte, Z. A., and Kellogg, C. A. (2013). Comparison of DNA preservation methods for environmental bacterial community samples. FEMS Microbiol. Ecol. 83, 468-477. doi: 10.1111/1574-6941.12008

Guo, F., and Zhang, T. (2013). Biases during DNA extraction of activated sludge samples revealed by high throughput sequencing. Appl. Microbiol. Biotechnol. 97, 4607-4616. doi: 10.1007/s00253-012-4244-4

Hall, A. B., Tolonen, A. C., and Xavier, R. J. (2017). Human genetic variation and the gut microbiome in disease. Nat. Rev. Genet. 18, 690-699. doi: 10.1038/nrg. 2017.63 
Hotamisligil, G. S. (2006). Inflammation and metabolic disorders. Nature 444, 860-867.

Kopylova, E., Noé, L., and Touzet, H. (2012). SortMeRNA: fast and accurate filtering of ribosomal RNAs in metatranscriptomic data. Bioinformatics 28, 3211-3217. doi: 10.1093/bioinformatics/bts611

Langille, M. G. I., Zaneveld, J., Caporaso, J. G., McDonald, D., Knights, D., Reyes, J. A., et al. (2013). Predictive functional profiling of microbial communities using 16S rRNA marker gene sequences. Nat. Biotechnol. 31, 814-821. doi: $10.1038 /$ nbt.2676

Ley, R. E., Peterson, D. A., and Gordon, J. I. (2006). Ecological and evolutionary forces shaping microbial diversity in the human intestine. Cell 124, 837-848. doi: $10.1016 /$ j.cell.2006.02.017

Lisko, D. J., Johnston, G. P., and Johnston, C. G. (2017). Effects of dietary yogurt on the healthy human gastrointestinal (GI) microbiome. Microorganisms 5:E6. doi: 10.3390/microorganisms5010006

Lozupone, C. A., Hamady, M., Kelley, S. T., and Knight, R. (2007). Quantitative and qualitative beta diversity measures lead to different insights into factors that structure microbial communities. Appl. Environ. Microbiol. 73, 1576-1585. doi: 10.1128/aem.01996-06

Lozupone, C. A., and Knight, R. (2015). The UniFrac significance test is sensitive to tree topology. BMC Bioinformatics 16:211. doi: 10.1186/s12859-015-0640-y

Macfarlane, S., and Macfarlane, G. T. (2003). Regulation of short-chain fatty acid production. Proc. Nutr. Soc. 62, 67-72. doi: 10.1079/pns2002207

Marco, M. L., Heeney, D., Binda, S., Cifelli, C. J., Cotter, P. D., Foligné, B., et al. (2017). Health benefits of fermented foods: microbiota and beyond. Curr. Opin. Biotechnol. 44, 94-102. doi: 10.1016/j.copbio.2016.11.010

May, L. A. (2011). Saline-Saturated DMSO-EDTA as A Storage Medium for Microbial DNA Analysis from Coral Mucus Swab Samples. Silver Spring: NOAA.

Penas-Steinhardt, A., Barcos, L. S., Belforte, F. S., de Sereday, M., Vilariño, J., Gonzalez, C. D., et al. (2012). Functional characterization of TLR4 3725 G/C polymorphism and association with protection against overweight. PLoS One 7:e50992. doi: 10.1371/journal.pone.0050992

Petruzzelli, M., and Moschetta, A. (2010). Intestinal ecology in the metabolic syndrome. Cell Metab. 11, 345-346. doi: 10.1016/j.cmet.2010.04.012

Rodríguez-Carrio, J., López, P., Sánchez, B., González, S., Gueimonde, M., Margolles, A., et al. (2017). Intestinal dysbiosis is associated with altered shortchain fatty acids and serum-free fatty acids in systemic lupus erythematosus. Front. Immunol. 8:23. doi: 10.3389/fimmu.2017.00023

Roesch, L. F. W., Casella, G., Simell, O., Krischer, J., Wasserfall, C. H., Schatz, D., et al. (2009). Influence of fecal sample storage on bacterial community diversity. Open Microbiol. J. 3, 40-46. doi: 10.2174/1874285800903010040

Rognes, T., Flouri, T., Nichols, B., Quince, C., and Mahé, F. (2016). VSEARCH: a versatile open source tool for metagenomics. PeerJ. 4:e2584. doi: 10.7717/peerj. 2584

Schmidt, M. I., Duncan, B. B., Vigo, A., Pankow, J. S., Couper, D., Ballantyne, C. M., et al. (2006). Leptin and incident type 2 diabetes: risk or protection? Diabetologia 49, 2086-2096. doi: 10.1007/s00125-006-0351-z

Schnorr, S. L., Candela, M., Rampelli, S., Centanni, M., Consolandi, C., Basaglia, G., et al. (2014). Gut microbiome of the Hadza hunter-gatherers. Nat. Commun. 5:3654. doi: $10.1038 /$ ncomms4654

Schultze, A., Akmatov, M. K., Andrzejak, M., Karras, N., Kemmling, Y., Maulhardt, A., et al. (2014). Comparison of stool collection on site versus at home in a population-based study. Bundesgesundheitsblatt - Gesundheitsforschung Gesundheitsschutz. 57, 1264-1269. doi: 10.1007/s00103-014-2051-z
Segata, N., Izard, J., Waldron, L., Gevers, D., Miropolsky, L., Garrett, W. S., et al. (2011). Metagenomic biomarker discovery and explanation. Genome Biol. 12:R60. doi: 10.1186/gb-2011-12-6-r60

Sinha, R., Chen, J., Amir, A., Vogtmann, E., Shi, J., Inman, K. S., et al. (2016). Collecting fecal samples for microbiome analyses in epidemiology studies. Cancer Epidemiol. Biomark. Prev. 25, 407-416. doi: 10.1158/1055-9965.EPI-150951

Sommer, F., Anderson, J. M., Bharti, R., Raes, J., and Rosenstiel, P. (2017). The resilience of the intestinal microbiota influences health and disease. Nat. Rev. Microbiol. 15, 630-638. doi: 10.1038/nrmicro.2017.58

Surana, N. K., and Kasper, D. L. (2017). Moving beyond microbiome-wide associations to causal microbe identification. Nature 552, 244-247. doi: 10.1038/ nature25019

Suzuki, Y., Ikeda, K., Sakuma, K., Kawai, S., Sawaki, K., Asahara, T., et al. (2017). Association between yogurt consumption and intestinal microbiota in healthy young adults differs by host gender. Front. Microbiol. 8:847. doi: 10.3389/fmicb. 2017.00847

Tercera encuesta nacional de factores de riesgo para enfermedades no transmisibles (2013). Tercera Encuesta Nacional de Factores de Riesgo Para Enfermedades No Transmisibles. Available at: http://www.msal.gob.ar/images/stories/bes/ graficos/0000000544cnt-2015_09_04_encuesta_nacional_factores_riesgo.pdf.

The Human Microbiome Project Consortium (2012). Structure, function and diversity of the healthy human microbiome. Nature 486, 207-214. doi: 10.1038/ nature 11234

Ussar, S., Griffin, N. W., Bezy, O., Fujisaka, S., Vienberg, S., Softic, S., et al. (2015). Interactions between gut microbiota, host genetics and diet modulate the predisposition to obesity and metabolic syndrome. Cell Metab. 22, 516-530. doi: 10.1016/j.cmet.2015.07.007

van den Elsen, L. W., Poyntz, H. C., Weyrich, L. S., Young, W., and Forbes-Blom, E. E. (2017). Embracing the gut microbiota: the new frontier for inflammatory and infectious diseases. Clin. Transl. Immunol. 6:e125. doi: 10.1038/cti.2016.91

Vogtmann, E., Chen, J., Amir, A., Shi, J., Abnet, C. C., Nelson, H., et al. (2017). Comparison of collection methods for fecal samples in microbiome studies. Am. J. Epidemiol. 185, 115-123. doi: 10.1093/aje/kww177

Voigt, A. Y., Costea, P. I., Kultima, J. R., Li, S. S., Zeller, G., Sunagawa, S., et al. (2015). Temporal and technical variability of human gut metagenomes. Genome Biol. 16:73. doi: 10.1186/s13059-015-0639-8

Wesolowska-Andersen, A., Bahl, M. I., Carvalho, V., Kristiansen, K., SicheritzPontén, T., Gupta, R., et al. (2014). Choice of bacterial DNA extraction method from fecal material influences community structure as evaluated by metagenomic analysis. Microbiome 2:19. doi: 10.1186/2049-2618-2-19

Conflict of Interest Statement: The authors declare that the research was conducted in the absence of any commercial or financial relationships that could be construed as a potential conflict of interest.

Copyright (๔) 2019 Belforte, Fernandez, Tonín Monzón, Rosso, Quesada, Cimolai, Millán, Cerrone, Frechtel, Burcelin, Coluccio Leskow and Penas-Steinhardt. This is an open-access article distributed under the terms of the Creative Commons Attribution License (CC BY). The use, distribution or reproduction in other forums is permitted, provided the original author(s) and the copyright owner(s) are credited and that the original publication in this journal is cited, in accordance with accepted academic practice. No use, distribution or reproduction is permitted which does not comply with these terms. 